\title{
Visual Assessment by Seasoned Operators versus Fractional Flow Reserve Guided Stenting in Patients with Multivessel Disease in Indian Patients
}

Vikrant Vijan*, Anjith Vupputuri, Manav Aggarwal, Sanjeev Chintamani, Bishnu Kiran Rajendran, Gurpreet Singh, Muthiah Subramanian and Rajesh Thachathodiyl

Department of Cardiology, Amrita Institute of Medical Sciences, Amrita Vishwa Vidyapeetam University, Kochi, Kerala, India

\begin{abstract}
Background: The benefit of revascularization is less clear in stenotic lesions that do not induce ischemia and medical therapy alone is likely to be equally effective. FFR (Fractional Flow Reserve) identifies stenoses that are causing reversible ischemia and thereby the operator can guide interventions to the lesions responsible for the patient's problem, saving time, cost and optimizing clinical outcome. The main aim is to compare FFR and visual assessment with respect to decision making, requirements of stents and corresponding outcomes in patients with multivessel coronary artery disease.

Method: This was a prospective, observational, single centre study, which included 38 patients randomized into 2 groups: one group that underwent FFR guided stenting for the borderline lesions, if the FFR value was found to be significant and for the other group the decision to go ahead with stenting for borderline lesions was based on independent opinion of 4 cardiologists participating in the study, based on visual assessment. The patients were followed up at 3 and 6 months. The primary endpoint of the study was death due to any cause or ACS (Acute Coronary Syndrome). The number of stents saved, cost savings and symptomatic improvement were the secondary outcomes that were studied. Inter-observer variation within the 4 operators in both the FFR and visual arms was also analysed.

Results: There were no deaths or ACS during 3 and 6 months follow-up in the 38 patients that were included in the two groups. There was no statistical difference with regards to number of stents saved, cost savings, and functional outcomes such as angina; which were our secondary end points between the visual assessment by seasoned operators and FFR guided stenting groups. There was inter-observer variation between all the 4 operators in our study with regards to visual assessment of the borderline lesions.
\end{abstract}

Conclusion: FFR is important in decision making in borderline lesions and it should be used more often, especially where there are single operators.

Keywords: FFR; Multivessel coronary artery disease; Percutaneous coronary intervention

\section{Introduction}

The presence of MI (Myocardial Infarction) is an important risk factor for adverse clinical outcomes in patients with coronary artery disease [1-3]. Revascularization of stenotic coronary lesions that induce ischemia can improve a patient's functional status and outcome [3-5]. For stenotic lesions that do not induce ischemia, however, the benefit of revascularization is less clear, and medical therapy alone is likely to be equally effective $[6,7]$.

In the current era of drug-eluting stents, the percentage of patients with multivessel coronary artery disease in whom PCI (Percutaneous Coronary Intervention) is performed has increased [8,9]. Because the drug-eluting stents are expensive and are associated with potential late complications, their appropriate and judicious use is critical and very important $[10,11]$. It is difficult, in patients with multivessel coronary artery disease, to determine which lesions cause ischemia and warrant stenting. Currently available non-invasive stress imaging modalities are limited in their ability to accurately localize ischemia-producing lesions in these patients [12].

Although coronary angiography is the standard technique for guiding PCI in patients with multivessel coronary disease, it often underestimates or overestimates a lesion's functional severity $[13,14]$. FFR (Fractional Flow Reserve) is a pressure derived, lesion specific, physiological index determining the hemodynamic severity of intracoronary lesions. FFR identifies stenosis that are causing reversible ischemia so that they can be treated, and helps eliminate stenosis that are not causing ischemia from consideration for treatment [15]. Using FFR, the operator can guide interventions to the lesions responsible for the patient's problem, saving time, cost and optimizing clinical outcome [16].

FFR is measured by placing a pressure guidewire across the lesion of interest and pharmacologically inducing hyperemia. The ratio of distal to proximal pressure allows the physician to physiologically determine if the narrowing is the cause of ischemia. It can be easily measured during coronary angiography by calculating the ratio of distal coronary pressure measured with a coronary pressure guidewire to aortic pressure measured simultaneously with the guiding catheter. An FFR value of 0.80 or less identifies ischemia-causing coronary stenosis with an accuracy of more than $90 \%$ [17-19].

*Corresponding author: Vikrant Vijan, MD, DM, Consultant Interventional Cardiologist, Department of Cardiology, Amrita Institute of Medical Sciences , Amrita Vishwa Vidyapeetam University, Kochi, Kerala-682 041, India, Tel: 91 9539681750; E-mail: vikrantvijan@hotmail.com

\section{Received March 30, 2016; Accepted May 04, 2016; Published May 09, 2016}

Citation: Vijan V, Vupputuri A, Aggarwal M, Chintamani S, Rajendran BK, et al. (2016) Visual Assessment by Seasoned Operators versus Fractional Flow Reserve Guided Stenting in Patients with Multivessel Disease in Indian Patients. Cardiovasc Pharm Open Access 5: 179. doi:10.4172/2329-6607.1000179

Copyright: ( 2016 Vijan V, et al. This is an open-access article distributed under the terms of the Creative Commons Attribution License, which permits unrestricted use, distribution, and reproduction in any medium, provided the original author and source are credited. 
Citation: Vijan V, Vupputuri A, Aggarwal M, Chintamani S, Rajendran BK, et al. (2016) Visual Assessment by Seasoned Operators versus Fractional Flow Reserve Guided Stenting in Patients with Multivessel Disease in Indian Patients. Cardiovasc Pharm Open Access 5: 179. doi:10.4172/2329-6607.1000179

Page 2 of 5

This study evaluates visual assessment by multiple operators guided stenting vs. FFR guided stenting and their outcomes. Thus, for patients with multivessel coronary artery disease identifying an approach to PCI that results in more judicious use of stents, while still achieving complete relief of myocardial ischemia, could improve clinical outcomes and decrease health care costs.

\section{Methodology}

In this prospective study, we included 38 patients having multivessel disease (one vessel had significant stenosis and other vessel/ vessels were borderline). Patients were randomized into 2 groups: one group underwent FFR guided stenting for all the borderline lesion/s and for the other group the decision to go ahead with stenting for borderline lesion/s was based on independent opinion of 4 cardiologists participating in the study, based on visual assessment. In the FFR arm, those patients with FFR value of less than or equal to 0.8 were advised stenting. All the patients in the FFR arm (whether underwent stenting or advised medical management) were followed up in the study. All the patients in the visual assessment group (whether underwent stenting or advised medical management) were followed up in the study. Both the groups of patients were followed up at 3 and 6 months (Figure 1).
In the FFR guided group, a RadiAnalyzer (St Jude Medical) and 0.014" diameter high fidelity pressure-recording wire (Pressure Wire, Radi Medical Systems, Uppsala, Sweden) was used for evaluation of lesion. Out of 19 patients in the FFR guided group, 12 patients received only intracoronary adenosine, 3 patients received only intravenous adenosine and there were 4 patients who received both intravenous as well as intracoronary adenosine for achievement of maximal hyperemia. Intracoronary adenosine boluses were administered in a dose of 90-120 $\mu \mathrm{g}$ in the right coronary artery, 60-180 $\mu \mathrm{g}$ in the left coronary artery and 120-180 $\mu \mathrm{g}$ in the left circumflex artery. Each bolus were followed by $5 \mathrm{ml}$ flush of normal saline as a transport medium and pressure signals during adenosine infusion and bolus injection would be recorded. The range of doses of intravenous adenosine was 90-180 $\mu \mathrm{g}$ in LAD and $120-140 \mu \mathrm{g}$ in LCX.

\section{Study endpoints}

The prime end point of the study was death due to any cause or acute coronary syndrome (unstable angina, non-ST segment elevation MI and ST- segment elevation MI). The secondary end point of the study was number of stents saved, cost savings and symptomatic improvement. Inter-observer variation within the 4 operators in visual arm was also analysed.

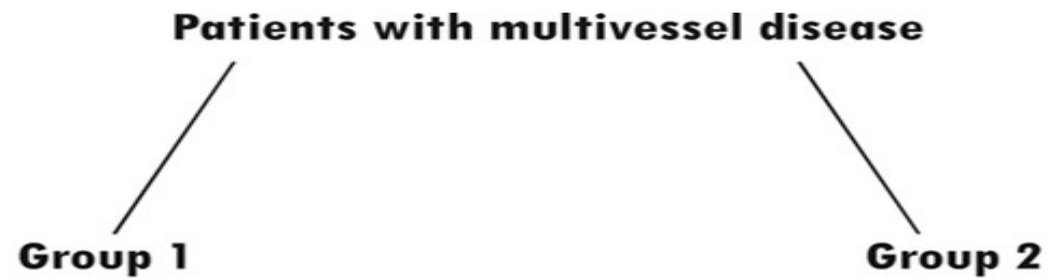

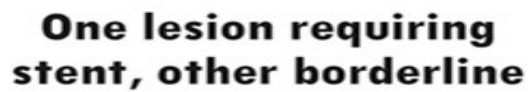
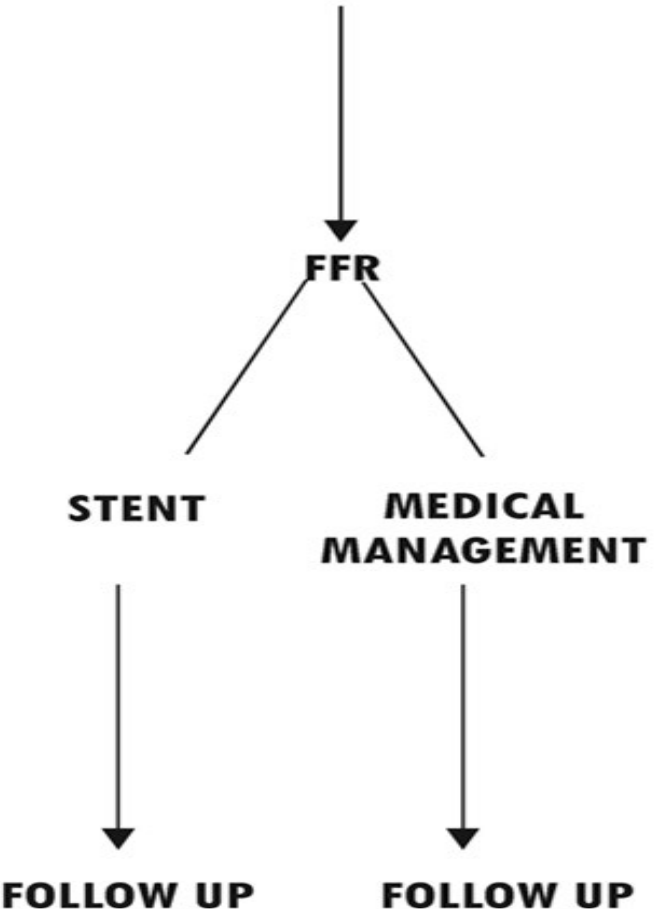

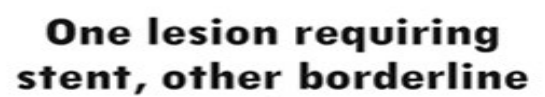

stent, other borderline

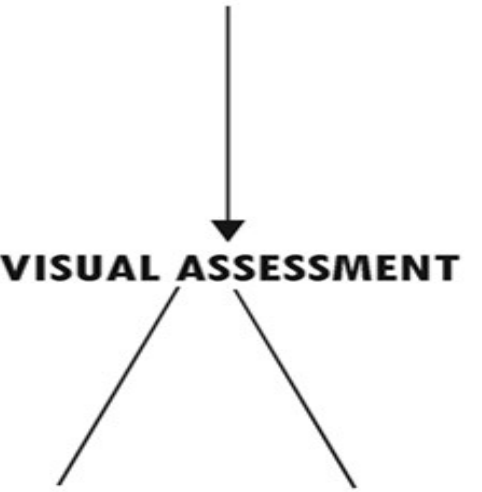

STENT

MEDICAL MANAGEMENT

Figure 1: Study design. 
Citation: Vijan V, Vupputuri A, Aggarwal M, Chintamani S, Rajendran BK, et al. (2016) Visual Assessment by Seasoned Operators versus Fractional Flow Reserve Guided Stenting in Patients with Multivessel Disease in Indian Patients. Cardiovasc Pharm Open Access 5: 179. doi:10.4172/2329-6607.1000179

Page 3 of 5

\section{Study population}

Patients were included if 1 ) age $\geq 20$ years 2 ) stable angina, unstable angina, NSTEMI, Recent awSTEMI (anterior wall ST elevation myocardial infarction) - atypical chest pain or no chest pain but with documented silent ischemia 3) multivessel disease with one significant lesion and other borderline lesion 4) eligibility for PCI.

Patients were excluded if 1) preferred treatment is CABG 2) left main coronary artery disease requiring revascularization 3 ) extremely tortuous or calcified coronary arteries precluding FFR measurements 4) multivessel disease with all lesions severely stenosed by visual arm

\section{Statistical analysis}

Continuous variable are expressed as mean and standard deviation whereas categorical variable are expressed as frequency and percentage. The Shapiros-Wilk's test was used for assessing the normality of the data. The variables between the groups are analyzed using chi-square test or Fisher's exact test based on the observations. A two sided $P$ value $<0.05$ was considered as statistically significant.

\section{Results}

\section{Baseline and lesion characteristics}

A total of 38 patients were included and followed up in the study. There were 19 patients in the FFR arm and 19 patients in the visual arm. There were 21 borderline lesions in the FFR arm and 20 borderline lesions in the visual arm.

Of the 19 patients in FFR arm, the mean age was $58.8 \pm 8.7$ years, whereas the mean age in the visual arm was $62.2 \pm 10.7$ years. Both the groups were equally matched with no significant statistical difference $(P=0.294)$. In the FFR arm, $73.7 \%$ of patients were males in contrast to $84.2 \%$ males in the visual arm. Thus, majority of the patients in our study were males and the groups were equally matched $(P=0.693)$. The detailed baseline and lesion characteristics of the patients are shown in Tables 1 and 2 .

In the FFR arm, there were 5 patients, who had significant FFR value and they underwent stenting whereas in the visual arm, 8 lesions out of the 20 borderline lesions underwent direct stenting based on visual assessment by multiple operators. The characteristics of these lesions are shown in Table 3. It was found that in the both the groups majority of the patients had Type B lesions and FFR arm had more proximal LAD (Left Anterior Descending) lesions (4 out of the 5 borderline lesions in the FFR ARM)

\section{Primary and secondary outcomes}

Primary outcomes of the study-There were no incidence of death or ACS during 3 and 6 months follow up period. Secondary outcomes of the study-Out of 5 lesions with significant FFR value, 3 borderline lesions underwent angioplasty with stents. The remaining 2 lesions, which were significant proximal LAD lesions, the patients underwent CABG (Coronary Artery Bypass Grafting) based upon the physician's discretion. Hence for statistical analysis, we considered that these 2 patients underwent stenting (1 stent each).

The 16 borderline lesions with non-significant FFR value were left on medical follow up. There was one patient in our study in the FFR arm, who had 2 significant lesions and 1 borderline lesion on visual assessment. Upon doing FFR, we found all three to be insignificant. Patient was thus left only on medical management. On follow up at 3 and 6 months, he was angina free and did not have any other complaints.
In the visual arm, 12 borderline lesions were kept on medical follow up.

For analysis of secondary outcomes, the number of stents used in borderline lesions in both the groups and the follow-up of all patients at 3 and 6 months were compared. In the FFR arm 5 stents were used in the 21 borderline lesions as compared to 8 stents in the 20 borderline lesions in the visual arm. There was no statistical difference between number of stents used in both the groups $(P=0.265)$. Thus, there was no significant difference in cost savings in both the groups.

All the patients in both the arms were followed up at 3 and 6 months for evaluating symptomatic improvement or occurrence of angina. In the FFR arm only one patient had occurrence of class II effort angina, however he was found to have anemia (Hemoglobin-8.3 g/dl) and he was symptomatically better after correction of anemia. The remaining 18 patients were angina free at 3 and 6 months follow-up. In the 19 patients in the visual arm, none of the patients had angina at the end of 3 and 6 months.

We also compared interobserver differences within the 4 operators of our study on basis of visual assessment of lesions in all the 38 patients (Table 4). After statistically analysis, it was found that there was interobserver variation between all the 4 operators in the study.

\section{Discussion}

Acute Myocardial Infarction (AMI) is one of the most commonly diagnosed conditions in hospitalized patients with chest pain. The culprit vessel is usually perceptible in patients with ST-elevation and acute coronary occlusion. In multivessel coronary artery disease, it is difficult to assess which lesions are associated with reversible ischemia and should be stented.

\begin{tabular}{|c|c|c|c|}
\hline Characteristics & FFR arm $(\mathbf{n = 1 9})$ & Visual arm $(\mathbf{n = 1 9 )}$ & $\boldsymbol{P}$-value \\
\hline Age (Mean \pm SD) & $58.8 \pm 8.7$ & $62.2 \pm 10.7$ & 0.294 \\
\hline Male & $73.7 \%$ & $84.2 \%$ & 0.693 \\
\hline Hypertension & $36.8 \%$ & $42.1 \%$ & 0.740 \\
\hline Diabetes Mellitus & $57.9 \%$ & $47.4 \%$ & 0.516 \\
\hline Dyslipidemia & $68.4 \%$ & $84.2 \%$ & 0.447 \\
\hline Stents used & 5 & 8 & 0.265 \\
\hline
\end{tabular}

Table 1: Baseline demographic characteristics of the patients in FFR arm and visual arm.

\begin{tabular}{|c|c|c|}
\hline Lesion location and type & $\begin{array}{c}\text { FFR arm } \\
(n=21 \text { lesions) }\end{array}$ & $\begin{array}{c}\text { Visual arm } \\
\text { ( } n=20 \text { lesions) }\end{array}$ \\
\hline \multicolumn{3}{|l|}{ Lesion location } \\
\hline Proximal LAD & 7 & 2 \\
\hline Mid LAD & 5 & 0 \\
\hline Diagonal & 1 & 3 \\
\hline Proximal LCx & 0 & 3 \\
\hline Mid LCx & 3 & 0 \\
\hline $\mathrm{OM}$ & 3 & 6 \\
\hline Mid RCA & 2 & 1 \\
\hline Distal RCA & 0 & 2 \\
\hline PDA & 0 & 3 \\
\hline \multicolumn{3}{|l|}{ ACC/AHA lesion classification } \\
\hline $\begin{array}{l}\text { Type A } \\
\text { (high success, >85\%; low risk) }\end{array}$ & 8 & 6 \\
\hline $\begin{array}{l}\text { Type B } \\
\text { (moderate success, } 60 \text { to } 85 \% \text {; } \\
\text { moderate risk) }\end{array}$ & 10 & 13 \\
\hline $\begin{array}{l}\text { Type C } \\
\text { (low success, }<60 \% \text {; high risk) }\end{array}$ & 3 & 1 \\
\hline
\end{tabular}

Table 2: Lesion characteristics of the patients in FFR arm and visual arm. 
Citation: Vijan V, Vupputuri A, Aggarwal M, Chintamani S, Rajendran BK, et al. (2016) Visual Assessment by Seasoned Operators versus Fractional Flow Reserve Guided Stenting in Patients with Multivessel Disease in Indian Patients. Cardiovasc Pharm Open Access 5: 179. doi:10.4172/2329-6607.1000179

Page 4 of 5

\begin{tabular}{|l|c|c|}
\hline \multicolumn{1}{|c|}{ Lesion location and type } & \multicolumn{1}{|c|}{$\begin{array}{c}\text { FFR arm (n=5 } \\
\text { lesions) }\end{array}$} & $\begin{array}{c}\text { Visual arm (n=8 } \\
\text { lesions) }\end{array}$ \\
\hline Lesion location & 4 & 1 \\
\hline Proximal LAD & 1 & 0 \\
\hline Mid LAD & 0 & 2 \\
\hline Diagonal & 0 & 0 \\
\hline Proximal LCx & 0 & 1 \\
\hline Mid LCx & 0 & 0 \\
\hline OM & 0 & 1 \\
\hline Mid RCA & 0 & 2 \\
\hline Distal RCA & 0 & 0 \\
\hline PDA & & 7 \\
\hline ACC/ AHA lesion classification & 2 & 1 \\
\hline $\begin{array}{l}\text { Type A } \\
\text { (high success, }>85 \% ; \text { low risk) }\end{array}$ & 3 & \\
\hline $\begin{array}{l}\text { Type B } \\
\text { (moderate success, } 60 \text { to } 85 \% ; \\
\text { moderate risk) }\end{array}$ & & \\
\hline $\begin{array}{l}\text { Type C } \\
\text { (low success, }<60 \% \text {; high risk) }\end{array}$ & & \\
\hline
\end{tabular}

Table 3: Lesion characteristics of the patients who underwent stenting

\begin{tabular}{|c|c|}
\hline Observers & $\boldsymbol{P}$-value \\
\hline Observers 1 and 2 & 0.478 \\
\hline Observers 1 and 3 & 0.355 \\
\hline Observers 1 and 4 & 0.492 \\
\hline Observers 2 and 3 & 0.062 \\
\hline Observers 2 and 4 & 0.552 \\
\hline Observers 3 and 4 & 0.207 \\
\hline
\end{tabular}

Table 4: Inter-observer variation between different operators in the study.

The reliability of FFR in assessing the hemodynamic significance of lesions in AMI and unstable angina has been demonstrated by few studies [20,21]. Fractional flow reserve versus Angiography for Multivessel Evaluation (FAME) study has evidenced that at 2 years, rates of death and myocardial infarction were significantly lower in FFR-guided group than in the angiography-guided PCI group [22]. Moreover, few studies have also shown that the use of FFR-guided PCI was cost saving $[23,24]$.

Our study had a distinct design. All the patients were randomly allocated to either the FFR arm or the visual arm. In the FFR arm, if the borderline vessel was found to be significant, the patient would be advised stenting. If FFR value was not significant, the patients would be recommended medical follow up. In the visual arm the decision to go ahead with stenting of borderline lesion/s was based on opinion of majority of operators participating in the study. If majority of the operators felt the borderline lesion was not significant, the patient would be advised medical follow up.

We had 4 operators whose independent opinions were taken in the study. Both these groups were followed up at 3 and 6 months. Inter-observer variation within the 4 operators in visual arm was also analysed and demonstrated that there was inter-observer variation between the operators. For a particular lesion, one of the observers would recommend that it is not significant enough to be tested further by FFR and could be left for medical management, whereas another observer would recommend the same lesion to be significant and he would like to go ahead with direct stenting for the same lesion. This underlines the limitations of visual assessment of severity of borderline lesions.

In one of the retrospective analysis, demonstrating clinical usefulness and cost effectiveness of fractional flow reserve among Indian patients (FIND study) it was found that concordance of management plan assessed by angiography alone and by angiography with FFR is about $58 \%$, which means that $>40 \%$ of intermediate lesions would be classified wrongly using angiography alone [25]. Despite of the proven fact that the measurement of FFR allows more judicious use of stents and maximizes the benefits of PCI by accurate discrimination of stenosis that benefit most from revascularization, the use of FFR, in decision making program is grossly underutilized. Usually, in our practice majority of the patients undergo decision to go ahead with stenting based on visual assessment of the lesions by a single operator.

To the best of our knowledge, such a study has not been conducted before in the Indian population. We tried to analyze whether visual assessment by multiple operators and their decision to guide stenting or to keep patient on medical follow up for borderline lesions is comparable to gold standard FFR. From the above statistics we can say visual assessment by operators can identify the borderline lesions and when multiple operator's opinion is taken their decision is comparable to the gold standard FFR with regards to the primary and secondary outcomes. But, it is not always possible to have multiple operators. Many a times the decision to go ahead with stenting for borderline lesions or to keep the patient on medical follow up is decided by a single operator.

In such cases wherever the lesion significance is doubtful and the lesion is of moderate severity FFR should be considered especially in centers, where a single operator is available.

\section{Study Limitations}

A large number of patients are needed to actually test the significance between the two groups with regards to number of stents and costs saved. Also, a long-term follow up would give a better idea regarding the importance of FFR in decision making in borderline lesions.

\section{Conclusion}

FFR is preferable for decision making in borderline lesions and should be used more often especially where there are single operators.

\section{References}

1. Beller GA, Zaret BL (2000) Contributions of nuclear cardiology to diagnosis and prognosis of patients with coronary artery disease. Circulation 101: 1465-1478.

2. Shaw LJ, Iskandrian AE (2004) Prognostic value of gated myocardial perfusion SPECT. J Nucl Cardiol 11: 171-185.

3. Shaw LJ, Berman DS, Maron DJ, Mancini GB, Hayes SW, et al. (2008) Optimal medical therapy with or without percutaneous coronary intervention to reduce ischemic burden: results from the Clinical Outcomes Utilizing Revascularization and Aggressive Drug Evaluation (COURAGE) trial nuclear sub-study. Circulation 117: 1283-1291.

4. Davies RF, Goldberg AD, Forman S, Pepine CJ, Knatterud GL, et al. (1997) Asymptomatic Cardiac Ischemia Pilot (ACIP) study two-year follow-up: outcomes of patients randomized to initial strategies of medical therapy versus revascularization. Circulation 95: 2037-2043.

5. Erne $P$, Schoenenberger AW, Burckhardt D, Zuber M, Kiowski W, et al (2007) Effects of percutaneous coronary interventions in silent ischemia after myocardial infarction: the SWISSI II randomized controlled trial. Jama 297:1985-1991.

6. Boden WE, O'Rourke RA, Teo KK, Hartigan PM, Maron DJ, et al. (2007) Optimal medical therapy with or without $\mathrm{PCl}$ for stable coronary disease. $\mathrm{N}$ Engl J Med 356: 1503-1516. 
Citation: Vijan V, Vupputuri A, Aggarwal M, Chintamani S, Rajendran BK, et al. (2016) Visual Assessment by Seasoned Operators versus Fractional Flow Reserve Guided Stenting in Patients with Multivessel Disease in Indian Patients. Cardiovasc Pharm Open Access 5: 179. doi:10.4172/2329-6607.1000179

Page 5 of 5

7. Pijls NH, van Schaardenburgh P, Manoharan G, Boersma E, Bech JW, et al. (2007) Percutaneous coronary intervention of functionally non-significant stenosis: 5 year follow-up of the DEFER Study. J Am Coll Cardiol 49: 21052111.

8. Moses JW, Stone GW, Nikolsky E, Mintz GS, Dangas G, et al. (2006) Drugeluting stents in the treatment of intermediate lesions: pooled analysis from four randomized trials. Journal of the American College of Cardiology 47: 21642171.

9. Ong AT, van Domburg RT, Aoki J, Sonnenschein K, Lemos PA, et al. (2006) Sirolimus-eluting stents remain superior to bare-metal stents at two years: medium-term results from the Rapamycin-Eluting Stent Evaluated at Rotterdam Cardiology Hospital (RESEARCH) registry. Journal of the American College of Cardiology 47: 1356-1360.

10. Kaiser C, Brunner-La Rocca HP, Buser PT, Bonetti PO, Osswald S, et al (2005) Incremental cost-effectiveness of drug-eluting stents compared with a third-generation bare-metal stent in a real-world setting: randomised Base Stent Kosten Effektivitäts Trial (BASKET). The Lancet 366: 921-929.

11. Pfisterer M, Brunner-La Rocca HP, Buser PT, Rickenbacher $P$, Hunziker $P$, et al. (2006) Late clinical events after clopidogrel discontinuation may limit the benefit of drug-eluting stents: an observational study of drug-eluting versus bare-metal stents. Journal of the American College of Cardiology 48: 25842591.

12. Lima RS, Watson DD, Goode AR, Siadaty MS, Ragosta M, et al. (2003) Incremental value of combined perfusion and function over perfusion alone by gated SPECT myocardial perfusion imaging for detection of severe threevessel coronary artery disease. Journal of the American College of Cardiology 42: 64-70.

13. Fischer JJ, Samady H, McPherson JA, Sarembock IJ, Powers ER, et al. (2002) Comparison between visual assessment and quantitative angiography versus fractional flow reserve for native coronary narrowings of moderate severity. The American journal of cardiology 90: 210-215.

14. Topol EJ, Nissen SE (1995) Our preoccupation with coronary luminology the dissociation between clinical and angiographic findings in ischemic heart disease. Circulation 92: 2333-2342.

15. De Bruyne B, Sarma J (2008) Fractional flow reserve: a review: invasive imaging. Heart 94: 949-959.
16. Tonino PA, De Bruyne B, Pijls NH, Siebert U, Ikeno F, et al. (2009) Fractiona flow reserve versus angiography for guiding percutaneous coronary intervention. N Engl J Med 360: 213-224.

17. Pijls NH, Van Gelder B, Van der Voort P, Peels K, Bracke FA, et al. (1995) Fractional flow reserve. A useful index to evaluate the influence of an epicardial coronary stenosis on myocardial blood flow. Circulation 92: 3183-3193.

18. De Bruyne B, Pijls NH, Bartunek J, Kulecki K, Bech JW, et al. (2001) Fractional flow reserve in patients with prior myocardial infarction. Circulation 104: 157162.

19. Pijls NH, De Bruyne B, Peels K, Van Der Voort PH, Bonnier HJ, et al. (1996) Measurement of fractional flow reserve to assess the functional severity of coronary-artery stenoses. N Engl J Med 334: 1703-1708.

20. Fischer JJ, Wang XQ, Samady H, Sarembock IJ, Powers ER, et al. (2006) Outcome of patients with acute coronary syndromes and moderate coronary lesions undergoing deferral of revascularization based on fractional flow reserve assessment. Catheterization and cardiovascular interventions 68: 544548

21. Potvin JM, Rodés-Cabau J, Bertrand OF, Gleeton O, Nguyen CN, et al. (2006) Usefulness of fractional flow reserve measurements to defer revascularization in patients with stable or unstable angina pectoris, non-ST-elevation and STelevation acute myocardial infarction, or atypical chest pain. Am J Cardiol 98: 289-297.

22. Pijls NH, Fearon WF, Tonino PA, Siebert U, Ikeno F, et al. (2010) Fractional flow reserve versus angiography for guiding percutaneous coronary intervention in patients with multivessel coronary artery disease: 2 year follow-up of the FAME (Fractional Flow Reserve Versus Angiography for Multivessel Evaluation) study. Journal of the American College of Cardiology 56: 177-184.

23. Fearon WF, Bornschein B, Tonino PA, Gothe RM, De Bruyne B, et al. (2010) Economic evaluation of fractional flow reserve-guided percutaneous coronary intervention in patients with multivessel disease. Circulation 122: 2545-2550.

24. Murphy JC, Hansen PS, Bhindi R, Figtree GA, Nelson GI, et al. (2014) Cost benefit for assessment of intermediate coronary stenosis with fractional flow reserve in public and private sectors in Australia. Heart Lung Circ 23: 807-810.

25. Sengottuvelu G, Chakravarthy B, Rajendran R, Ravi S (2014) Clinical usefulness and cost effectiveness of fractional flow reserve among Indian patients (FIND study). Catheter Cardiovasc Interv. 